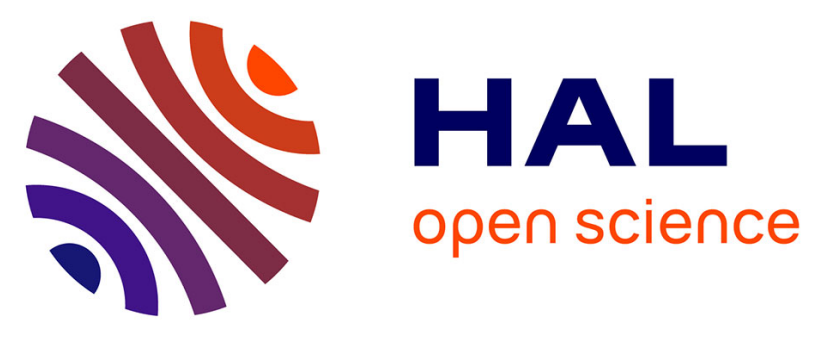

\title{
Miniaturization of Transmission Lines: Meandered Slow-wave CPWs
}

\author{
Marc Margalef-Rovira, Tadeu Mota Frutuoso, Abdelhalim Saadi, Loïc \\ Vincent, Manuel J. Barragan, Emmanuel Pistono, Christophe Gaquière, \\ Philippe Ferrari
}

\section{To cite this version:}

Marc Margalef-Rovira, Tadeu Mota Frutuoso, Abdelhalim Saadi, Loïc Vincent, Manuel J. Barragan, et al.. Miniaturization of Transmission Lines: Meandered Slow-wave CPWs. IEEE MTT-S International Conference on Microwaves for Intelligent Mobility (ICMIM 2020), Nov 2020, Linz (virtual), Austria. 10.1109/ICMIM48759.2020.9299107 . hal-03371412

\section{HAL Id: hal-03371412 \\ https://hal.science/hal-03371412}

Submitted on 11 Oct 2021

HAL is a multi-disciplinary open access archive for the deposit and dissemination of scientific research documents, whether they are published or not. The documents may come from teaching and research institutions in France or abroad, or from public or private research centers.
L'archive ouverte pluridisciplinaire HAL, est destinée au dépôt et à la diffusion de documents scientifiques de niveau recherche, publiés ou non, émanant des établissements d'enseignement et de recherche français ou étrangers, des laboratoires publics ou privés.

\section{(ㄷ)(1) $\$$}

Distributed under a Creative Commons Attribution - NonCommerciall 4.0 International 
This is the author's version of an article that has been published in this conference. Changes were made to this version by the publisher prior to publication. The final version of record is available at http://dx.doi.org/10.1109/ICMIM48759.2020.9299107

\section{Miniaturization of Transmission Lines: Meandered Slow-wave CPWs}

\author{
Marc Margalef-Rovira \\ Université Grenoble Alpes, CNRS, \\ Grenoble INP*, TIMA F-38000 \\ Université Grenoble Alpes, Grenoble \\ INP*, RFIC-Lab F-38000 Grenoble, \\ France \\ marc.margalef-rovira@univ-grenoble- \\ alpes.fr \\ Loic Vincent \\ Université Grenoble Alpes, Grenoble \\ INP*, F-38000 \\ Grenoble, France \\ loic.vincent@grenoble-inp.fr \\ Christophe Gaquiere \\ Université des Sciences et Technologies \\ de Lille, IEMN F-60069 \\ Villeneuve d'Asq, France \\ christophe.gaquiere@univ-lille.fr
}

\author{
Tadeu Mota-Frutuoso \\ Université Grenoble Alpes, Grenoble \\ INP*, RFIC-Lab F-38000 Grenoble, \\ France \\ Universidãe de São Paulo \\ São Paulo, Brasil \\ tadeu.mota-frutuoso@univ-grenoble- \\ alpes.fr
}

\author{
Manuel J. Barragan \\ Université Grenoble Alpes, CNRS, \\ Grenoble INP*, TIMA F-38000) \\ Grenoble, France \\ manuel.barragan@univ-grenoble- \\ alpes.fr
}

Philippe Ferrari

Université Grenoble Alpes, Grenoble

INP*, RFIC-Lab F-38000 Grenoble,

France

philippe.ferrari@univ-grenoble-alpes.fr

\author{
Abdelhalim A. Saadi \\ Université Grenoble Alpes, Grenoble \\ INP*, RFIC-Lab F-38000 Grenoble, \\ France \\ ahmed-abdelhalim.saadi@univ- \\ grenoble-alpes.fr
}

\author{
Emmanuel Pistono \\ Université Grenoble Alpes, Grenoble \\ INP*, RFIC-Lab F-38000 Grenoble, \\ France \\ emmanuel.pistono@univ-grenoble- \\ alpes.fr
}

\begin{abstract}
This work presents novel transmission line structures based on Meandered Slow-wave CoPlanar Waveguides (MS-CPWs), aiming to achieve high miniaturization. As a proof-of-concept, these two kinds of transmission lines were designed and fabricated in the AMS $0.35 \mu \mathrm{m}$ CMOS technology together with classical straight and meandered microstrip lines. Measurement results from $70 \mathrm{kHz}$ to $100 \mathrm{GHz}$ of the fabricated transmission lines are presented. At $80 \mathrm{GHz}$, all the considered transmission lines present similar quality factors, ranging between 10 and 14 . On the other hand, at this frequency, one of the developed MS-CPW presents an effective dielectric constant of 88, while the meandered microstrip exhibits an equivalent effective dielectric constant of 51, thus leading to higher compactness for the MS-CPW.
\end{abstract}

Keywords-transmission line, slow-wave, $C P W$, mm-wave

\section{INTRODUCTION}

Current and future use of transceivers for diverse applications such as Internet of Things (IoT), automotive radar, 5G (future 6G) or medical applications require to operate in several frequency bands and the use of diverse communication standards. In the best scenario, the change of such telecommunication standard can be achieved through the operation of tunable elements in the transceiver. However, changing the telecommunication standard under use often means switching complete parts of the transceiver or even using a completely different transceiver.

In this scenario, the downscaling of the transistor size plays a major role in the miniaturization of complex transceivers. However, miniaturization of passive devices, needed to perform filtering, impedance matching or beamsteering functions, is a less technology-dependent issue. Passive devices are divided into two families: (i) lumped elements and (ii) distributed elements. Lumped elements (i.e. capacitors, inductors and transformers) tend to have a high Quality-factor (Q-factor) at RF frequencies (i.e. around 10 $\mathrm{GHz}$ ) that decreases with the increase of frequency, while distributed elements (i.e. transmission lines) tend to have a low Q-factor at RF frequencies that increases with the frequency. Moreover, the physical length of a transmission line (TLine) with a given electrical length is inversely proportional to the frequency. For these reasons, lumped elements are preferred at low frequencies (below $\sim 30-50 \mathrm{GHz}$ in integrated technology). Above these frequencies, TLines are preferred thanks to their increased performance and acceptable size.

TLine footprint reduction has been addressed in the literature using two main approaches: (i) meandering [1,2] or (ii) using slow-wave concept [3]. In 2003, the first proposal of the application of the slow-wave effect on CPWs was published [3], presenting the so-called Slow-wave CPW structure (S-CPW). In 2013, the first proposal of the distributed electrical model for S-CPWs was published [4] and subsequently improved in [5]. In [5], authors provide tools for the analytical modeling of this structure, allowing the designer to accurately simulate the S-CPW in any technology, greatly reducing the effort to be devoted to time-consuming EM simulations.

In this work, innovative structures aiming to reduce the footprint of CoPlanar Waveguides (CPWs) are proposed. The proposed structures utilize the meandering and the slow-wave concept to reduce their physical length per unit of electrical length. Recently, meandering techniques together with the slow-wave concept was proposed for slow-wave microstrips, as in [6]. However, to the best of author's knowledge, this is the first time that an approach merging both techniques is proposed for CPW structures.

This work is organized as follows: section II provides a brief explanation of the slow-wave concept. In section III, the proposed structures are presented. Section IV presents the results of the fabricated TLines. Finally, section $\mathrm{V}$ presents the main conclusions of this work. 


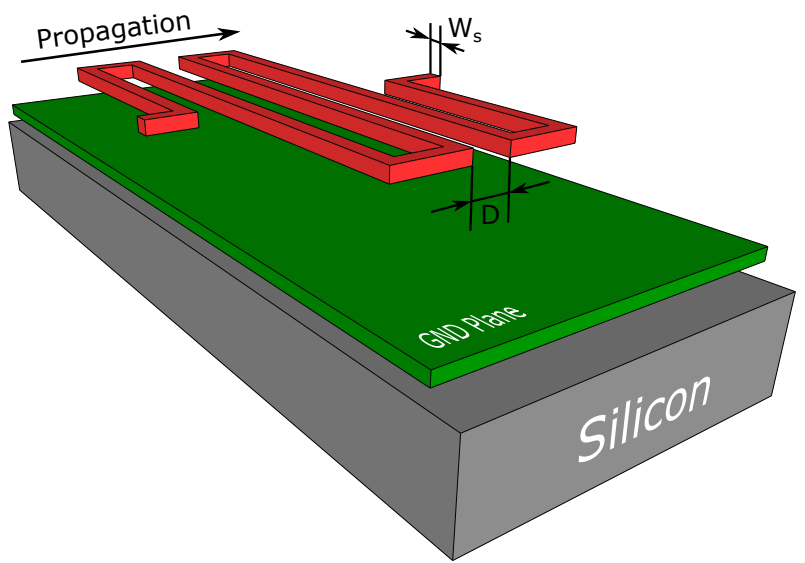

(a)

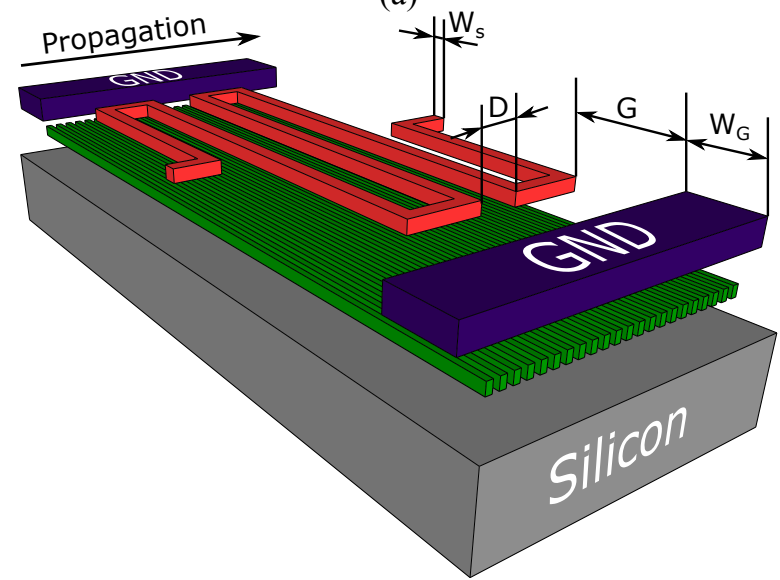

(b)

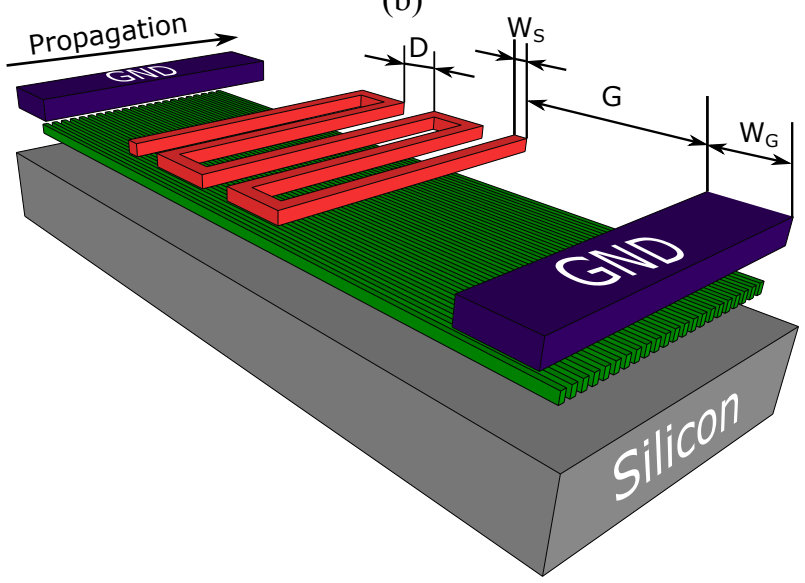

(c)

Fig. 1 (a) Meandered microstrip. (b) Meandered S-CPW type 1. (c) Meandered S-CPW type 2

\section{The SlOW-Wave PrinciPle}

The slow-wave concept has been applied to multiple TLine architectures such as: microstrip [6], CoPlanar Waveguides (CPWs) [3,4,5], and CoPlanar Striplines (CPS) [7]. This technique utilizes some patterned structures to capture the electrical field of the TLine while allowing the magnetic field to flow freely. From a circuit point of view, this results in an increase of the capacitance per unit of length of the Tline, $C_{\text {lin }}$, and an do not modify inductance per unit of length, $L_{\text {lin }}$. The increase of $C_{\text {lin }}$ leads to a reduction of the propagation velocity $\left(v_{\varphi}\right)$, whose expression is given by:

$$
v_{\varphi}=\frac{1}{\sqrt{C_{\text {lin }} \cdot L_{\text {lin }}}}
$$

Hence, the physical length $(L)$ of a TLine, with a given electrical length $(\theta)$, is reduced when the slow-wave concept is used since:

$$
\theta=\frac{\omega}{v_{\varphi}} \cdot L
$$

where $\omega$ is the considered angular frequency.

In addition, the Q-factor of a TLine can be expressed as:

$$
Q=\frac{\omega}{2 \cdot v_{\varphi} \cdot \alpha}
$$

where $\alpha$ is the attenuation constant of the considered TLine. Using this expression, it can be proved that the use of the slowwave concept can lead to an improved Q-factor as compared to the classical structures.

In practice, the slow-wave concept for coplanar structures is achieved through the implementation of a floating shield beneath the signal and ground strips. This floating shield is composed of narrow metal ribbons placed transversally to the propagation direction.

\section{Proposed ARCHITECTURE}

This work explores the effect of meandering the signal strip of S-CPWs. Note that two kinds of structures can be envisioned with this consideration: (i) a structure where most of the signal strips are placed transversally to the overall propagation direction, or (ii) a structure where most of the signal strips are placed in parallel to the overall propagation direction. In this paper, these kinds of Meandered S-CPWs (MS-CPWs) are named type 1 and 2, respectively.

Fig. 1(a) presents a classical meandered microstrip line and the geometrical parameters for its design. In this work, together with a classical microstrip, this structure will be used as a reference for the evaluation of the performance of the proposed MS-CPWs. Figs. 1(b) and 1(c) show 3D views of the so-called type 1 and type $2 \mathrm{MS}-\mathrm{CPWs}$ and their geometrical parameters.

In a traditional meandered microstrip line, whether the strip is meandered transversally or parallelly to the overall propagation direction has a negligible effect on the performance of the TLine. However, the behavior of the type 1 or type 2 MS-CPWs is slightly different.

Both of the proposed MS-CPW types present two propagation modes within the structure. A first mode where the propagation occurs as in a S-CPW. This mode is found for the regions where the signal strip is placed parallelly to the overall propagation direction. A second mode of propagation is found for the regions where the signal strip is placed transversally to the overall propagation direction. In this case, the propagation mode is similar to that of a microstrip line. Even though the electrical field is still captured by the shield below, the signal strip is parallel to the metallic ribbons. Hence, return-current path is created in the metallic ribbons. Thus, behaving like a degenerated microstrip line where the return path for the signal is done through the metallic ribbons, with a capacitance existing between the signal strip and the metallic ribbons, and an inductance given by the flux of the magnetic field around the strips. As a result, type $1 \mathrm{MS}-\mathrm{CPW}$ are expected to show a lower propagation constant as compared to their type 2 counterparts. 


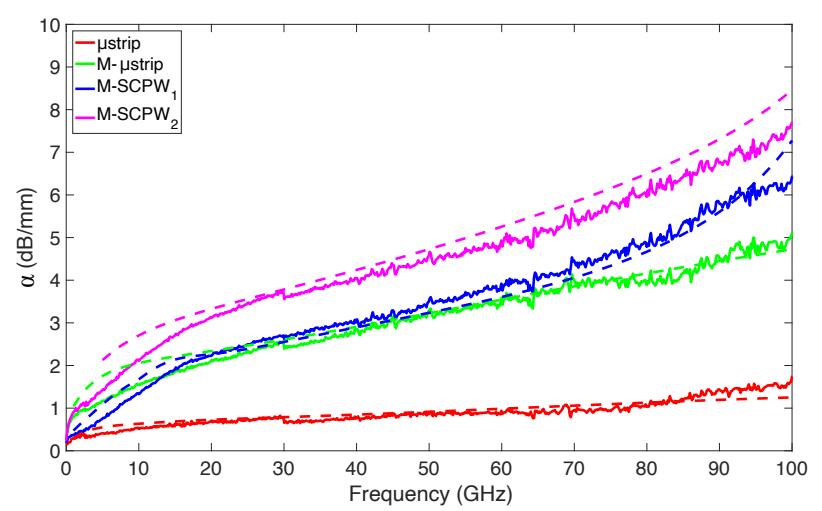

(a)

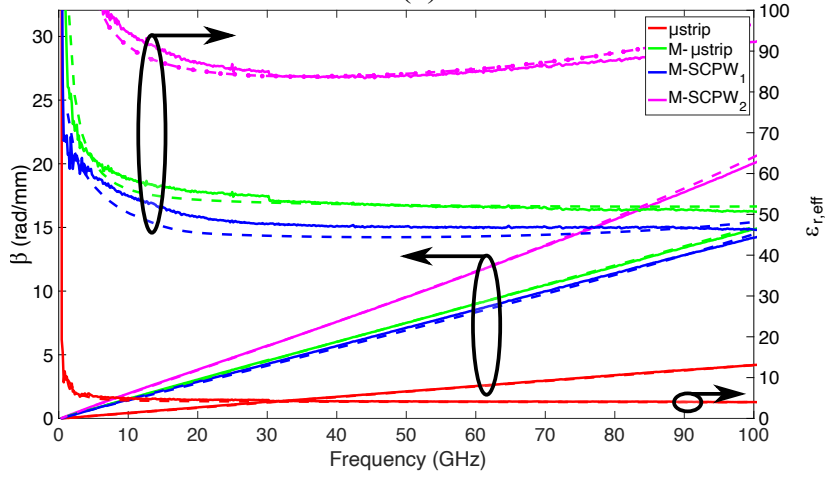

(b)

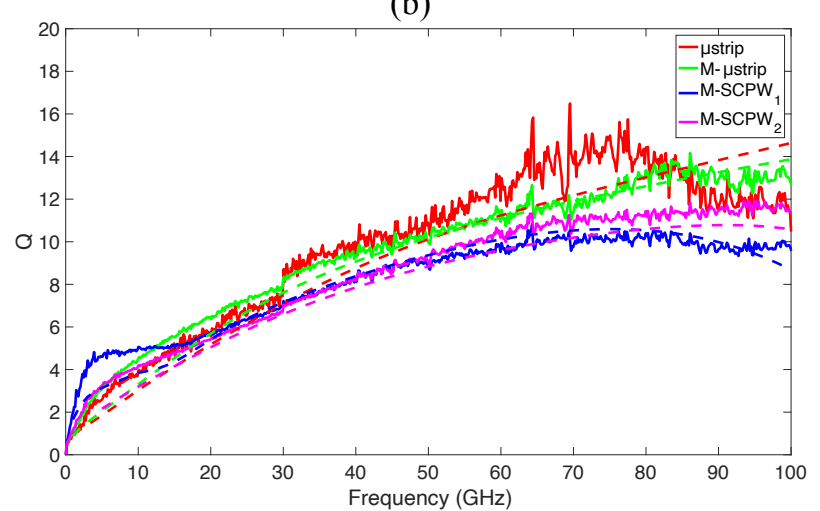

(c)

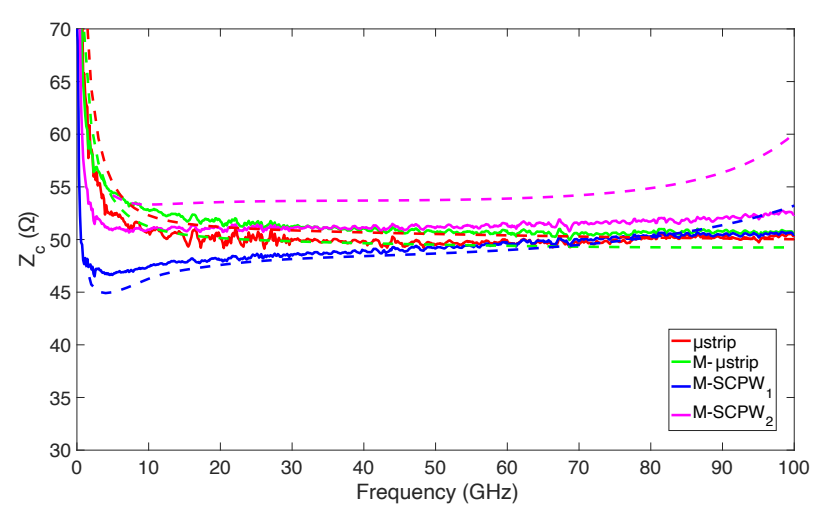

(d)

Fig. 2 Measurement (solid lines) and simulation results (dashed lines) of the considered TLines. (a) Attenuation constant. (b) Propagation constant and effective dielectric constant (c) $Q$ factor. (d) Characteristic impedance.

\section{RESULTS}

As a proof-of-concept, four different Tlines were designed, fabricated and measured on the AMS $0.35 \mu \mathrm{m}$ CMOS technology. The selected architectures were: (i) a 50$\Omega$ traditional microstrip line, (ii) a $50-\Omega$ meandered microstrip, (iii) a $50-\Omega$ type $1 \mathrm{MS}-\mathrm{CPW}$, and (iv) a $50-\Omega$ type 2 MS-CPW. Measurements were performed using an Anritsu VectorStar ME7838A4, from $70 \mathrm{kHz}$ to $100 \mathrm{GHz}$, with a Line-Reflect-Reflect-Line (LRRM) [8] calibration on an Impedance Standard Substrate (ISS). Subsequently, the effect of the pads and the 200- $\mu \mathrm{m}$ access lines were de-embedded using the technique described in [9].

For a fair comparison, all the circuits were allocated in an area of $120 \mu \mathrm{m}$ of length and $132 \mu \mathrm{m}$ of width. In particular, meandered microstrip TLines were designed with a margin of $20 \mu \mathrm{m}$ in each of their sides due to the fact that in a practical case any circuit placed inside this spatial margin would suffer from electromagnetic coupling. On the other hand, the confined electrical field of S-CPW-based TLines allows to place circuitry close to the TLine, while avoiding cross-talk issues.

The used technology features 3 metal layers and an optional additional thick layer, which was used for the strips design. In this technology, aluminum is the metal of choice for the Back-End-Of-Line (BEOL). It is well-known that the conductivity of aluminum is sensibly lower than the conductivity of copper. Hence, better results are expected in a technology featuring a copper BEOL.

The microstrip line was designed with a width of $5.5 \mu \mathrm{m}$ using the top thick metal for the signal strip and metal 1 for the ground plane. The meandered microstrip line was designed using the same metal layers and width than the classical microstrip and a spacing between parallel lines, $D$, of $11 \mu \mathrm{m}$, to avoid self-coupling between meandered strips. The type 1 MS-CPW was designed using metal 4 for the signal and ground strips and metal 3 for the floating ribbons. The width of the ground, $W_{G}$, and signal strips, $W_{S}$, was set to $8 \mu \mathrm{m}$ and $10 \mu \mathrm{m}$, respectively. The distance between signal and ground strips, $G$, was set to $13 \mu \mathrm{m}$. Finally, the spacing between parallel lines, $D$, was designed using $19 \mu \mathrm{m}$. Concerning the type $2 \mathrm{MS}-\mathrm{CPW}$, the floating ribbons were placed in the metal 3 layer and the signal and ground strips in the metal 4 layer. $W_{s}$ and $W_{G}$ were set to $5.5 \mu \mathrm{m}$ and $16 \mu \mathrm{m}$, respectively. The distance between the signal and ground strips, $G$, was designed using $13 \mu \mathrm{m}$. The distance between parallel strips, $D$, was set to $12 \mu \mathrm{m}$. Finally, for both MSCPWs the width and spacing of the floating ribbons was set to 0.6 and $2.6 \mu \mathrm{m}$, respectively.

Measurement and simulation are shown in Fig. 2 using solid and dashed lines, respectively. The presented results show very good agreement throughout the considered band. Fig. 2(a) shows the attenuation constant, $\alpha$, throughout the considered frequency band of the measured TLines. At 80 $\mathrm{GHz}$, the traditional microstrip presents an attenuation constant of around $1.3 \mathrm{~dB} / \mathrm{mm}, \alpha$ is equal to $4 \mathrm{~dB} / \mathrm{mm}$ for the meandered microstrip, around $5 \mathrm{~dB} / \mathrm{mm}$ for the MS-CPW type 1 , and around $6.6 \mathrm{~dB} / \mathrm{mm}$ for the MS-CPW type, respectively.

Fig. 2(b) presents the propagation constant and effective dielectric constant, $\epsilon_{\text {reff }}$, from $70 \mathrm{kHz}$ to $100 \mathrm{GHz}$ for the considered TLines. At $80 \mathrm{GHz}$, the classical microstrip 
presents a $\beta$ and an $\epsilon_{\text {reff }}$ of $3.5 \mathrm{rad} / \mathrm{mm}$ and 4.2, respectively. The meandered microstrip has a $\beta$ and an $\epsilon_{\text {reff }}$ of $12 \mathrm{rad} / \mathrm{mm}$ and 51 , respectively. The type 1 and 2

MS-CPWs present a $\beta$ of around 11.5 and 16, respectively. Finally, these two TLines present an $\epsilon_{\text {reff }}$ of 47 and 88, respectively.

Note that the values presented in Figs. 2(a) and 2(b) express magnitudes per unit of length. These values were obtained using the effective length of the structure, $120 \mu \mathrm{m}$.

Figure 2(c) presents the Q-factor of the considered Tlines. At $80 \mathrm{GHz}$, the microstrip and meandered microstrip present a Q-factor of 14 and 13, respectively. At the same frequency, the type 1 and type $2 \mathrm{MS}-\mathrm{CPW}$ s present Q-factors of 12 and 10 , respectively.

Figure 2(d) presents the characteristic impedance, $Z_{c}$, of the considered TLines throughout the analyzed frequency band, with all of them achieving the desired $50 \Omega$. Note that the type $2 \mathrm{MS}-\mathrm{CPW}$ shows a resonance at around $120 \mathrm{GHz}$. This is due to the fact that, at this frequency this TLine reaches 180 degrees of electrical length.

Finally, Table I summarizes the performance of the considered Tlines at $80 \mathrm{GHz}$. A Figure-of-Merit (FoM) is introduced, aiming to compare the miniaturization capabilities of the presented TLines. The considered FoM is obtained through the ratio between the electrical length of the TLine at the considered frequency and the occupied surface, which is equal to $0.016 \mathrm{~mm}^{2}$ for all the considered TLines. For the microstrip and meandered microstrip, the FoM has a value of 26 and $91 \mathrm{rad} / \mathrm{mm}^{2}$, respectively. The FoM has a a value of 87 and $120 \mathrm{rad} / \mathrm{mm}^{2}$ for the type 1 and type $2 \mathrm{MS}$ CPWs, respectively.

Table I. TLines PeRformance COMPARISON AT $80 \mathrm{GHz}$

\begin{tabular}{|c|c|c|c|c|}
\hline TLine Type & $\mathrm{Q}$ & $\operatorname{Zc}(\Omega)$ & $\frac{\theta}{S}\left(\mathrm{rad} / \mathrm{mm}^{2}\right)$ & $\epsilon_{\text {reff }}$ \\
\hline Microstrip & 14 & 50 & 26 & 4 \\
\hline $\begin{array}{c}\text { Meandered } \\
\text { Microstrip }\end{array}$ & 13 & 50 & 91 & 51 \\
\hline $\begin{array}{c}\text { MS-CPW } \\
\text { Type 1 }\end{array}$ & 12 & 50 & 87 & 47 \\
\hline $\begin{array}{c}\text { MS-CPW } \\
\text { Type 2 }\end{array}$ & 10 & 52 & 120 & 88 \\
\hline
\end{tabular}

\section{CONCLUSIONS}

In this work, four 50- $\Omega$ transmission line architectures were compared: (i) a straight microstrip line, (ii) a meandered microstrip line, (iii) a type $1 \mathrm{MS}-\mathrm{CPW}$ and (iv) a type $2 \mathrm{MS}-$ CPW. The presented measurements, from $70 \mathrm{kHz}$ to $100 \mathrm{GHz}$, show the capability of the type $2 \mathrm{MS}-\mathrm{CPW}$ to achieve high miniaturization. At $80 \mathrm{GHz}$, this TLine shows an $\epsilon_{\text {reff }}$ of 88, greater than the $\epsilon_{\text {reff }}$ shown by the meandered microstrip or the type $1 \mathrm{M}-\mathrm{SCPW}$, which is of 51 and 47 , respectively. In addition, measurement results of the fabricated TLines show their equivalent performance in terms of Q-factor, with values ranging from 14 to 10 at $80 \mathrm{GHz}$.

Together with the presented TLines, other TLines were sent into fabrication to carry out a comparison between the synthesizable characteristic impedance in this technology by using these architectures. In addition, microstrip lines and MS-CPWs were fabricated in a 55-nm technology featuring a copper BEOL, aiming to show the Q-factor improvement due to the increased conductivity. Future work includes the characterization of these TLines as well as the development of a parametric electrical model.

\section{ACKNOWLEDGMENT}

This work has been funded by the European Union (ECSEL JU GA 737454: TowARds Advanced BiCMOS NanoTechnology platforms for RF applicatiOns - Taranto), and the General Directorate for Enterprises (DGE) in France.

\section{REFERENCES}

[1] M. Kuhn, M. Klinger, A. Baranyak and J. H. Hinken, "HTSC inverted and conventional geometry microstrip resonator for UHF frequencies," in IEEE Transactions on Magnetics, vol. 27, no. 2, pp. 2809-2812, March 1991.

[2] Chih-Chiang Chen and C. -. C. Tzuang, "Synthetic quasi-TEM meandered transmission lines for compacted microwave integrated circuits," in IEEE Transactions on Microwave Theory and Techniques, vol. 52, no. 6, pp. 1637-1647, June 2004.

[3] T. S. D. Cheung, J. R. Long, K. Vaed, R. Volant, A. Chinthakindi, C. M. Schnabel, J. Florkey, and K. Stein, "On-chip interconnect for mmwave applications using an all-copper technology and wavelength reduction," in 2003 IEEE International Solid-State Circuits Conference (ISSC), 2003. Digest of Technical Papers. ISSCC., pp. 396-501 vol.1, Feb 2003.

[4] A. L. Franc, E. Pistono, G. Meunier, D. Gloria, and P. Ferrari, “A lossy circuit model based on physical interpretation for integrated shielded slow-wave cmos coplanar waveguide structures," IEEE Transactions on Microwave Theory and Techniques (T-MTT), vol. 61, no. 2, pp. 754-763, 2013.

[5] A. Bautista, A. L. Franc, and P. Ferrari, "Accurate Parametric Electrical Model for Slow-Wave CPW and Application to Circuits Design," IEEE Transactions on Microwave Theory and Techniques (TMTT), vol. 63, no. 12 , pp. 4225-4235, 2015.

[6] G. Acri, F. Podevin, E. Pistono, L. Boccia, N. Corrao, T. Lim, E. N. Isa, and P. Ferrari, "A Millimeter-Wave Miniature Branch-Line Coupler in 22-nm CMOS Technology," in IEEE Solid-State Circuits Letters, vol. 2, no. 6, pp. 45-48, June 2019.

[7] M. Abdel Aziz, H. Issa, D. Kaddour, F. Podevin, A.M.E. Safwat, E. Pistono, J.-M. Duchamp, A. Vilcot, J.-M. Fournier, and P. Ferrari "Shielded Coplanar Striplines for RF Integrated Applications", Microwave and Optical Technology Letters, Vol. 51, No. 1, pp. 352358, Feb. 2009

[8] A. Davidson et al., "LRM and LRRM Calibrations with Automatic Determination of Load Inductance," 36th ARFTG Conference Digest, Monterey, CA, USA, 1990, pp. 57-63.

[9] A. M. Mangan, S. P. Voinigescu, Ming-Ta Yang and M. Tazlauanu, "De-embedding transmission line measurements for accurate modeling of IC designs," in IEEE Transactions on Electron Devices, vol. 53, no. 2, pp. 235-241, Feb. 2006. 